\title{
Diffusion Coefficients of CdSe/CdS Quantum Rods in Water Measured Using Polarized Fluorescence Correlation Spectroscopy
}

\author{
Jaeran Lee ${ }^{1}$, Chan-Gi Pack ${ }^{2}$, Soo Yong $\mathrm{Kim}^{3}$, and Sok Won Kim ${ }^{1 *}$ \\ ${ }^{I}$ Department of Physics, University of Ulsan, Ulsan 680-749, Korea \\ ${ }^{2}$ Confocal Microscope Core Laboratory, Asan Institute for Life Sciences, Asan Medical Center, \\ 88, Olympic-ro 43-gil, Songpa-gu, Seoul 138-736, Korea \\ ${ }^{3}$ Department of Physics, Korea Advanced Institute of Science and Technology, Daejeon 305-701, Korea
}

(Received June 2, 2014 : revised September 2, 2014 : accepted September 19, 2014)

\begin{abstract}
A polarization fluorescence correlation spectroscopy system based on a confocal microscope was built to study the rotational and translational diffusion of $\mathrm{CdSe} / \mathrm{CdS}$ quantum rods (Q-rods), with the same and different polarization states between the polarizer and the analyzer (i.e. the $X X X$ and $X Y Y$ states). The rotational diffusion amplitude showed the dependences on polarization of $0.75 \pm 0.05$ in the $X X X$ state and $0.26 \pm 0.03$ in the $X Y Y$ state, when the translational diffusion amplitude was 1 . The diffusion coefficients of the Q-rods were found based on their translational and rotational diffusion times in the two polarization states, in solutions with viscosity ranging from 0.9 to $6.9 \mathrm{cP}$. The translational and rotational diffusion coefficients ranged from $1.5 \times 10^{-11}$ to $2.6 \times 10^{-12} \mathrm{~m}^{2} \mathrm{~s}^{-1}$ and from $2.9 \times 10^{5}$ to $5.6 \times 10^{4} \mathrm{~s}^{-1}$, respectively.
\end{abstract}

Keywords: Fluorescence correlation spectroscopy, Quantum rod, Photon counting

OCIS codes : (260.5430) Polarization; (170.6280) Spectroscopy, fluorescence and luminescence; (070.4550) Correlators

\section{INTRODUCTION}

Fluorescence correlation spectroscopy (FCS) was developed to study molecular diffusion dynamics and the photochemical properties of various fluorescent probes. FCS is based on the analysis of time-dependent fluorescence fluctuations from diffusing fluorescent probes. Since the late 1990s FCS has progressed due to advances in confocal systems and correlators, and due to the development of detectors with high quantum efficiency. It is well known that the diffusivity of a small particle in solution depends on the size of the particle and the viscosity of the solution. In most studies employing FCS, the relative size of the particle and viscosity of the sample solution have been determined using the Stokes-Einstein equation, based on the diffusion coefficient of a well-known probe in water [1-5]. These studies were unable to confirm particle shape, but the translational diffusion of fluorescent probes has often been investigated assuming that particles are spherical $[6,7]$.

The timescale of rotational diffusion for nanometer-sized probes introduces technical challenges, because the detectable time ranges of the correlator become expanded. However, photon antibunching and triplet formation occur on a timescale similar to that of rotational diffusion [8]. Fortunately, larger fluorescent probes (nanometer- or submicrometer-sized fluorescent particles such as quantum dots (Q-dots) and Q-rods) have different time scales for antibunching and lifetime, and they have no triplet state to complicate the analysis. Quantum particles are useful as probes due to their broad absorption bands, narrow emission bands, and high quantum yields $[9,10]$. In particular, Q-rods of well-defined size and shape can be used as a standard to verify experimentally the diffusion properties of various rodlike molecules and particles.

In this study we built a simple confocal-microscope-based polarization FCS system using CW-laser excitation to investigate the rotational and translational diffusion of $\mathrm{CdSe} / \mathrm{CdS}$ quantum rods. By autocorrelation analyses of various combinations of polarization and viscosity, we characterized the dependence of the rotational and translational diffusion of Q-rods on polarization by using the amplitude and characteristic time of the correlation function [11].

\footnotetext{
*Corresponding author: sokkim@ulsan.ac.kr

Color versions of one or more of the figures in this paper are available online.
} 


\section{THEORY}

In a confocal microscope system, the movements of fluorescent particles into and out of the focal volume cause the detected fluorescence to vary in intensity, and this intensity variation inherently contains information about the size, number, shape, and speed of the particles. It is difficult to confirm a large number of total-time series data varying over less than a few milliseconds, so instead these data may be analyzed via the following correlation function:

$$
G_{\text {cross }}(\tau)=\frac{\left\langle\delta F_{1}(t) \delta F_{2}(t+\tau)\right\rangle}{\left\langle F_{1}(t)\right\rangle\left\langle F_{2}(t)\right\rangle},
$$

where $\delta F_{1}(t)$ is the fluctuation deviation in fluorescence intensity on one detector at time $t, \delta F_{2}(t+\tau)$ is the fluctuation deviation in fluorescence intensity on the other detector at time $(t+\tau)$, and $\left\langle F_{1}(t)\right\rangle$ and $\left\langle F_{2}(t)\right\rangle$ are the average fluorescence intensities of the time series signals at each detector. In general the correlation function obtained from experimental measurements shows several exponential decays, including those due to antibunching, triplet state, and translational and rotational diffusions [8]. The well-known correlation model equation includes three terms representing translational diffusion $\left(G_{D}(\tau)\right)$, triplet state $\left(G_{T}(\tau)\right)$, and rotational diffusion $\left(G_{R}\right.$ $(\tau))$ :

$$
G(\tau)=G_{R}(\tau) \cdot G_{T}(\tau) \cdot G_{D}(\tau)
$$

This equation can be expanded as follows:

$$
\begin{aligned}
& G(\tau)=\left[1+R \exp \left(-\frac{\tau}{\tau_{R}}\right)\right] \cdot\left[1+\frac{F \exp \left(-\tau / \tau_{T}\right)}{1-F}\right] \\
& \cdot\left[G(0)\left(1+\left(\frac{\tau}{\tau_{D}}\right)^{\alpha}\right)^{-1}\left(1+\left(\left(\frac{w}{z}\right)^{2}\left(\frac{\tau}{\tau_{D}}\right)\right)^{\alpha}\right)^{-1 / 2}\right],
\end{aligned}
$$

where $R$ is the rotational fraction, $\tau_{R}$ is the rotational diffusion time, $F$ is the fraction of the triplet state, $\tau_{T}$ is the triplet state lifetime, $G(0)$ is the initial amplitude of translational diffusion, and $\tau_{D}$ is the translational diffusion time. The terms $\tau_{R}, \tau_{T}$, and $\tau_{D}$ are characteristic times corresponding to the FWHM of each region. The beam waist $w$ and axial length $z$ are related to the form of the confocal volume [12].

The third term of Eq. (3) has been well established by various experiments showing that the parameters of the translational diffusion term carry quantitative information about the average particle number contained in the focal volume during $\tau_{D}$, the diffusion coefficient, the hydrodynamic radius of the particles, and the viscosity of the solvent as determined by the Stokes-Einstein equation. The average particle number is simply the reciprocal of $G(0)$.
The second term indicating the triplet-state differs according to the presence or absence of each fluorophore. For fluorescent molecules such as rhodamine, this term varies according to background conditions [13], and this term is not present for semiconductors such as Q-dots. The first term shows a rotational diffusion; in the rotational diffusion, the rotational fraction $R$ varies according to the polarization state of the FCS system $[14,15]$. In FCS the rotational diffusion coefficient $D_{R}$ and translational diffusion coefficient $D_{T}$ are often used to compare the dynamical properties of particles, given by Eq. (4) [16]

$$
\begin{aligned}
D_{R} & =\frac{1}{6 \tau_{R}}: \text { rotational diffusion coefficient } \\
D_{T} & =\frac{\langle w\rangle^{2}}{4 \tau_{D}}: \text { translational diffusion coefficient }
\end{aligned}
$$

\section{EXPERIMENT}

\subsection{Optical Setup}

The FCS setup is illustrated schematically in Fig. 1. A 532-nm DPSS laser (Cobolt, Samba; $25 \mathrm{~mW}$ ) and a singlephoton counting module (SPCM; Id Quantique, id100-50) were used as the excitation source and detector respectively. Alignment of the optical components was accomplished using a commercial optical microscope (Olympus IX71). The optical components in the FCS setup were a polarization beam splitter (PBS), nonpolarized beam splitter (NPBS), excitation filter (ExF), dichroic mirror (DM), objective lens (OB), cover glass $(\mathrm{CG})$, emission filter $(\mathrm{EmF})$, lens $(\mathrm{L})$, mirror $(\mathrm{M})$, and pinhole (PH).

In this setup, the laser beam from the light source passes through the PBS and enters the optical microscope. The beam then passes through the ExF (Semrock, FF01-531/40-25) and reflects off the DM (Semrock, FF545/650-Di02). The reflected beam passes through the OB (Olympus, 60×, 1.2 NA), the immersion water, and the $\mathrm{CG}$, and then focuses on a very small volume of a few fL inside the solution. The fluorescent particles in the focal volume are excited by the laser beam and then emit fluorescent light; a portion of this light enters the OB. After the light passes through the DM, EmF, and $\mathrm{L}$, it escapes the microscope by the inner mirror. The light emerging from the microscope passes the $\mathrm{PH}$, which is $30 \mu \mathrm{m}$ in diameter, and the PBS. The PH and PBS are used to make a confocal conjugate point for the confocal volume, and to pass only the polarized fluorescence light, respectively. The light is split into two beams by the NPBS, and these beams are detected by two SPCMs. The fluorescent light detected at the SPCMs is converted into TTL signals, which are then converted into a correlation function by using a time-correlated single-photon counting system (PicoQuant, Picoharp300). The correlation function is monitored as the cross-correlation function of the responses 


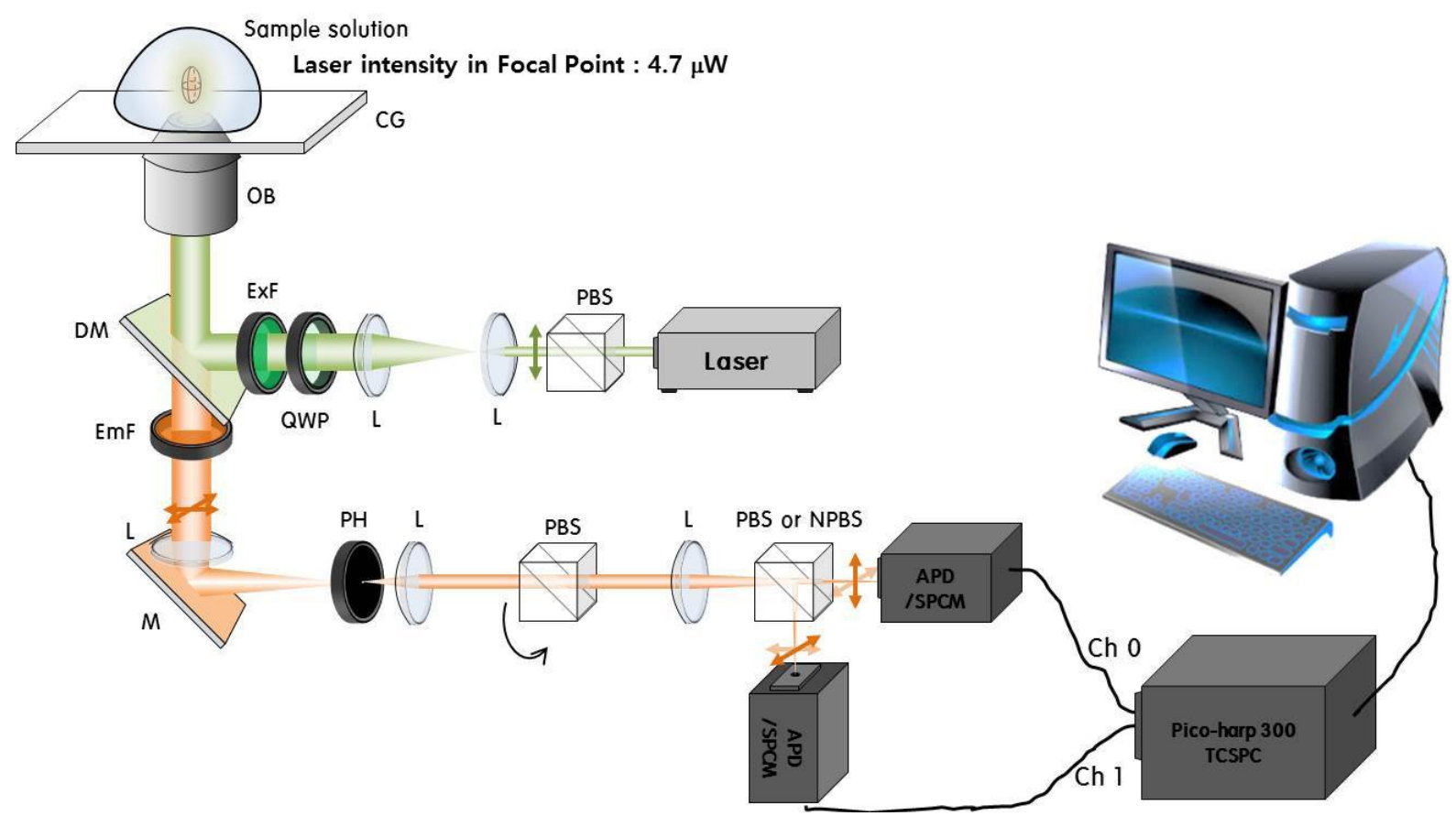

FIG. 1. The polarization FCS setup, based on an optical microscope.

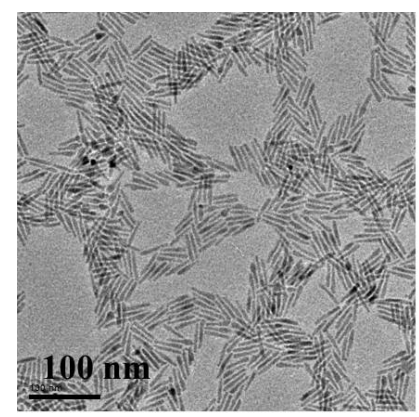

(a)

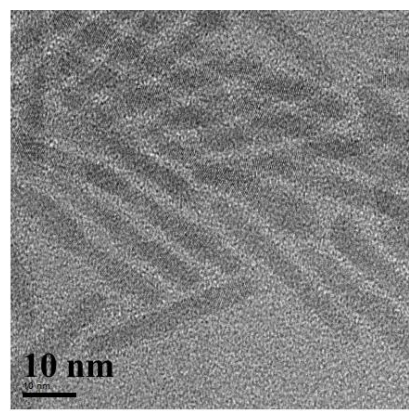

(b)
FIG. 2. (a) SEM and (b) TEM micrographs of $5 \mathrm{~nm} \times 35 \mathrm{~nm}$ (aspect ratio: 7) $\mathrm{CdSe} / \mathrm{CdS}$ Q-rods.

of the two SPCSMs, using the FluoFit program (PicoQuant) [16], the delay time of the correlation being limited to the range from $0.15 \mu \mathrm{s}$ to $0.1 \mathrm{~s}$.

\subsection{Preparation of Probe Sample}

To investigate the rotational diffusion of rodlike particles, we used Q-rods (Q-rod591) of length $35 \mathrm{~nm}$ and width 5 $\mathrm{nm}$ (aspect ratio of 7) as the fluorescent particles. SEM analysis showed that the Q-rods were of uniform high quality, and TEM analysis showed that they were of uniform size (Fig. 2). The Q-rods were of a core/shell configuration, with CdSe cores and CdS shells. Q-rod growth is described in detail in Ref. [17]. The Q-rods had a maximum emission peak at $591 \mathrm{~nm}$ and could be sufficiently excited by the 532-nm laser beam.

To characterize the rotational diffusion of the Q-rod591 particles, we diluted them to a concentration of a few $\mathrm{nM}$. At this concentration the number of Q-rods in the effective focal volume of our system would be less than 10 . Therefore, we prepared samples of two different concentrations such that the average particle number in the focal volume would be approximately 4 (the "high-concentration" solution) or 0.5 (the "low-concentration" solution). In addition, Q-rod solutions with various viscosities were prepared, to study the dependence of rotational diffusion on viscosity, and to allow a comparison of that result to the effect of translational diffusion on viscosity. Glycerin was used to adjust the viscosity of the solutions with volume percentages of 0 , $10,20,30,40$, and $50 \%$; the corresponding viscosities of these solutions at $23{ }^{\circ} \mathrm{C}$ were $0.9,1.2,1.7,2.6,4.0$, and $6.9 \mathrm{cP}$, as calculated using a formula for the viscosity of a glycerin-water mixture [18].

\subsection{Combination of Polarization States}

To examine the dependence of excitation and fluorescence on polarization states, two linear polarizers were used, one near the laser and one between the PH and the NPBS. The fluorescent light was split into two beams for cross correlation. The fluorescent light split by the NPBS was polarized into two states, $X X X$ and $X Y Y$, in which the first letter $X$ refers to the excitation light, and the last two letters denote the polarization states parallel to the excitation light $(X X)$ and perpendicular to the light $(Y Y)$, respectively.

\subsection{Effective Focal Volume of FCS System}

Before analyzing the diffusion of Q-rods, we confirmed the effective focal volume of the FCS system based on polari- 
zation states using a rhodamine $6 \mathrm{G}(\mathrm{R} 6 \mathrm{G})$ solution of about $10 \mathrm{nM}$ concentration. At our laser intensity the correlation curve of the R6G solution revealed a triplet state and a translational diffusion state. Thus, the curve was fitted by Eq. (3) assuming the triplet state and linear translational diffusion $(a=1)$. The fitting parameters was obtained via Eq. (3); for all polarization states the structure parameter $s=z / w$, triplet state time $\tau_{T}$, and initial amplitude of translational diffusion $G(0)$ were found to be $4.0 \pm 0.2,1.4 \pm 0.22$ $\mu \mathrm{s}$, and $0.57 \pm 0.03$ respectively, and the translational diffusion time $\tau_{D}$ was $43 \pm 4.7 \mu \mathrm{s}$ for the XXX state and $43 \pm 5.0 \mu \mathrm{s}$ for the XYY state. This result indicates that the effective focal volume ( $\left.V_{\text {eff }}\right)$ of our FCS system does not depend on the polarization state. The beam waist was calculated to be $0.266 \mu \mathrm{m}$ by Eq. (4), using the translational diffusion coefficient of R6G [19]. $V_{\text {eff }}$ was calculated from this result, using the equation $V_{\text {eff }}=\pi^{3 / 2} w^{2} z$, yielding a value of $0.42 \mathrm{fL}$ [1]. Additionally, to verify the performance of our FCS system we calculated the concentration of the R6G solution used in the measurements. The calculated concentration was $6 \mathrm{nM}$, which agreed fairly well with the expected value of $10 \mathrm{nM}$, with a difference of $4 \mathrm{nM}$; this indicated that the performance of the system was suitable to determine the concentration of the Q-rod solution. Therefore we determined the concentrations of both the low-concentration and highconcentration samples used in this study to be 2 and 16 $\mathrm{nM}$ respectively.

\section{RESULTS \& DISCUSSION}

To investigate the polarization dependence of the rotational diffusion of Q-rods, we built the polarization FCS system and measured correlation functions of the Q-rods' fluorescence responses. Measured functions were fitted by Eq. (3) to determine parameters related to the rotational and translational diffusion of the Q-rods. In Eq. (3) we neglected the triplet state because it does not appear in semiconductors such as Q-dots and Q-rods [19]. However, because the diffusion of Q-rods cannot be expected to be linear like that of Rhodamine 6G, due to the small differences in particle sizes and the irregular blinking in the full duration of the experiments, the anomalous diffusion exponent a was fitted by Eq. (3) using other parameters.

Before analyzing the influence of polarization, we examined the change in correlation function caused by varying the Q-rod concentration. Figure 3 shows the correlation functions obtained from Q-rod solutions of two different concentrations and the two solvent viscosities of 2.6 and $6.9 \mathrm{cP}$, in the $X X X$ polarization state. The correlation functions varied greatly with viscosity and sample concentration (Fig. 3(a)). In samples of viscosity $2.6 \mathrm{cP}$, the amplitude of the correlation function $G(\tau)$ increased as the sample concentration decreased. This result was also observed in samples of viscosity $6.9 \mathrm{cP}$, indicating that $G(\tau)$ is inversely proportional to the concentration of the solution. In general, the initial value of the translational diffusion $G(0)$ is proportional to the reciprocal of the number of particles in the effective focal volume. In the 2-nM samples, $G(\tau)$ increased as the viscosity of the sample was increased from 2.6 to 6.9 Q-rods' fluorescence response, but the variation in $G(\tau)$ was less than the concentration variation. A similar result was also obtained for the 16-nM samples, but the increase in $G(\tau)$ with viscosity was very small compared to that for the $2 \mathrm{nM}$ samples. This means that $G(\tau)$ depends more strongly on concentration than on viscosity. Although $G(\tau)$ showed a weaker dependence on viscosity than on concentration, the variation of $G(\tau)$ with viscosity cannot be neglected at the 2-nM concentration level.

As shown in Fig. 3(a), in samples of concentration 2 $\mathrm{nM}$ the $G(0)$ values of the correlation functions for viscosities of 2.6 and $6.9 \mathrm{cP}$ were 1.63 and 1.89 respectively. This result indicates that the effective focal volume was reduced as the viscosity of the solvent was increased. In both 2-nM samples the concentration of Q-rods was the same; therefore, the difference in viscosity was responsible for the variation in $G(0)$. The samples of viscosity $6.9 \mathrm{cP}$ used in this study included more glycerin than those of viscosity $2.6 \mathrm{cP}$; this increment of glycerin concentration increases the refractive index of the solution [20], thereby reducing the focal volume, and consequently decreasing the number of Q-rods passing through the reduced focal volume. This can be regarded as a reduction of the concentration of the solution.

According to the results in Fig. 3(a), the correlation functions obtained based on measurements of the Q-rod solutions were affected by the solutions' concentration and viscosity. As mentioned previously, the goal in this study is to investigate the rotational diffusion of Q-rods in combination with their polarization states. To carry this out, the influences of the concentration and the viscosity in the translational diffusion of the correlation function were eliminated by using a normalized correlation function. The normalization was achieved by dividing $G(\tau)$ by $G(0)$, which yielded the same normalized results for the two concentrations; the normalized functions differed only between the 2.6- and 6.9-cP viscosity groups (Fig. 3(b)). The function for the 6.9-cP group was timedelayed relative to that for the 2.6-cP group, but the normalized functions had the same amplitude in the rotational and translational diffusion regions, respectively. The functions were separated into regions corresponding to rotational and translational diffusion, described by two decay functions before and after approximately $10^{-5} \mathrm{~s}$. For translational diffusion the amplitude $G(0)$ was 1 , and the amplitude of rotational diffusion was $1.75 \pm 0.05$. The difference of the amplitudes of rotational diffusion and translational diffusion is the rotational fraction; in this case, the rotational fraction was $0.75 \pm 0.05$. This fraction has same value as the parameter $R$ obtained by fitting. At a viscosity of $2.6 \mathrm{cP}$, the initial value of the function was lower than the rotational fraction. This means that the rotational region begins before 0.15 $\mu \mathrm{s}$. 


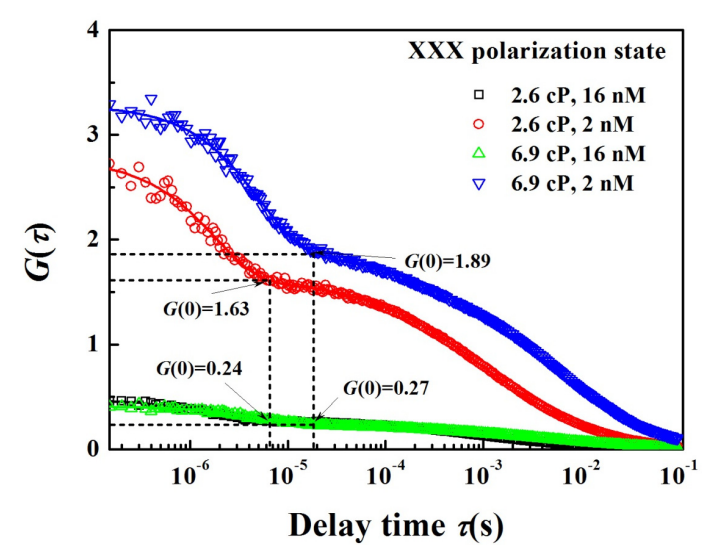

(a)

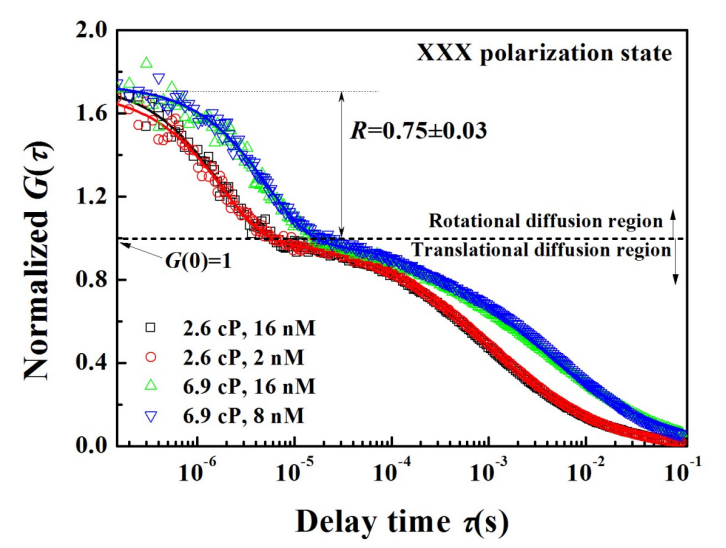

(b)

FIG. 3. (a) Fluorescence correlation functions for two viscosities and two particle concentrations; (b) normalized function of (a).

To analyze the rotational diffusion of Q-rods in combination with the polarization state, we measured and normalized the correlation function using viscosities of $0.9,1.2,1.73$, 2.57, 4.06, and $6.9 \mathrm{cP}$ and the polarization states $X X X$ and $X Y Y$, and the normalized functions were fitted by Eq. (3) to obtain the parameters $R, \tau_{R}, \tau_{D}$, and $\alpha$. For all polarization states the translational and rotational diffusion times increased as the viscosity increased. For the $X X X$ state, the rotational diffusion time increased from $0.58 \pm 0.055 \mu$ s to $5.17 \pm 0.50 \mu \mathrm{s}$ as the viscosity of the solvent increased from 0.9 to $6.9 \mathrm{cP}$; the diffusion time for the $X Y Y$ state showed a similar trend, with the rotational diffusion time increasing from $0.42 \pm 0.074 \mu$ s to $3.27 \pm 0.22 \mu$ s as the viscosity increased in the same range. For the lowest viscosity of $0.9 \mathrm{cP}$, the diffusion times of the $X X X$ and $X Y Y$ states differed slightly, by $0.16 \mu \mathrm{s}$, but for the viscosity of $6.9 \mathrm{cP}$ the difference was $1.90 \mu$ s. This result for the $6.9 \mathrm{cP}$ viscosity may be due to nonlinear diffusion caused by irregular blinking and the small differences in size of the Q-rods in the high-viscosity solvent. Furthermore, the anomalous diffusion exponent $a$, quantifying the nonlinear diffusion of the Q-rods, decreases from 0.75 to 0.61 with increasing solvent viscosity [21]. This result also may indicate that the difference in diffusion

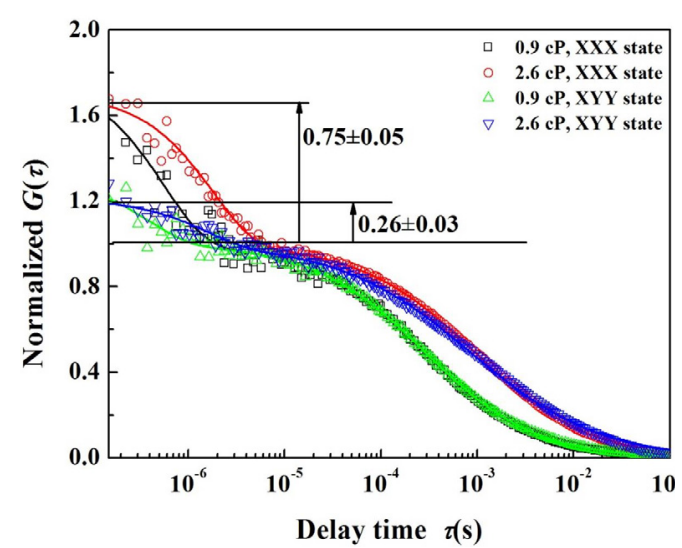

FIG. 4. Normalized fluorescence correlation functions for two viscosities and both the $X X X$ and $X Y Y$ states. Symbols and solid lines respectively denote measured and fitted data.

times under high-viscosity conditions is due not to the polarization state but to nonlinear diffusion [22].

Figure 4 illustrates the normalized $G(\tau)$ for viscosities of 0.9 and $2.6 \mathrm{cP}$ and the two polarization states studied; as shown in the figure, the rotational fractions were $0.75 \pm 0.05$ in the $X X X$ state and $0.26 \pm 0.03$ in the $X Y Y$ state. The fractions in the $X Y Y$ state were smaller than those in the $X X X$ state, which might an artifact of our FCS system. The system used in this study may be regarded as having three polarizers: a linear polarizer, a rotating linear polarizer, and an analyzer. The linear polarizer is in front of the light source, the rotating linear polarizer is the Q-rods in solution, and the linear analyzer is in front of the detector. The angle between the first polarizer and the last analyzer is $0^{\circ}$ in the $X X X$ state and $90^{\circ}$ in the $X Y Y$ state. The intensity of light passing through the three polarizers can be described by Malus' law [23]: At $0^{\circ}$ the maximum intensity ratio of input to output is 1 , and at $90^{\circ}$ the ratio is 0.25 . According to this assumption the fraction for the $X X X$ state would be 4 times as large as the value for the $X Y Y$ state, but the observed difference was about 3 times as large, indicating that the Q-rods do not act as an ideal linear polarizer. If the Q-rods were rotating linear polarizers then the rotational diffusion time would also be expected to change with polarization state, but the rotational axis in Brownian rotation of the rod like particles is random at the full duration of the experiments.

The rotational and translational diffusion coefficients were calculated by Eq. (4) using the diffusion time; the results are shown in Fig. 5(a) and Table 1. The results agree with the trend in diffusion time. As shown in Fig. 5a, the translational and rotational diffusion coefficients in $0.9-\mathrm{cP}$ samples were $(1.6 \pm 0.2) \times 10^{-11} \mathrm{~m}^{2} \mathrm{~s}^{-1}$ and $(2.9 \pm 0.4) \times 10^{5} \mathrm{~s}^{-1}$ for the $X X X$ state, and $(1.5 \pm 0.2) \times 10^{-11} \mathrm{~m}^{2} \mathrm{~s}^{-1}$ and $(3.9 \pm 0.6) \times 10^{5}$ $\mathrm{s}^{-1}$ for the $X Y Y$ state, respectively.

The relationship between $D_{R}$ and $D_{T}$ was fairly linear in all polarization states (Fig. 5(b)); the linear-fit values of the slope $D_{R} / D_{T}$ were $2.0 \times 10^{16} \mathrm{~m}^{-2}$ for the $X X X$ state and 
TABLE 1. Translational and rotational diffusion coefficients for various viscosities

\begin{tabular}{c|c|c|c|c|c|c}
\hline \hline$\eta(\mathrm{cP})$ & 0.89 & 1.22 & 1.73 & 2.57 & 4.06 & 6.88 \\
\hline$D_{T}\left(10^{-12} \mathrm{~m}^{2} \mathrm{~s}^{-1}\right)$ & $15.6 \pm 2.3$ & $11.1 \pm 1.4$ & $7.5 \pm 0.7$ & $4.9 \pm 0.2$ & $2.6 \pm 0.1$ & $1.6 \pm 0.05$ \\
\hline$D_{R}\left(10^{4} \mathrm{~s}^{-1}\right)$ & $29.9 \pm 3.8$ & $22.2 \pm 2.1$ & $14.6 \pm 0.8$ & $9.7 \pm 0.6$ & $5.2 \pm 0.4$ & $3.2 \pm 0.3$ \\
\hline
\end{tabular}

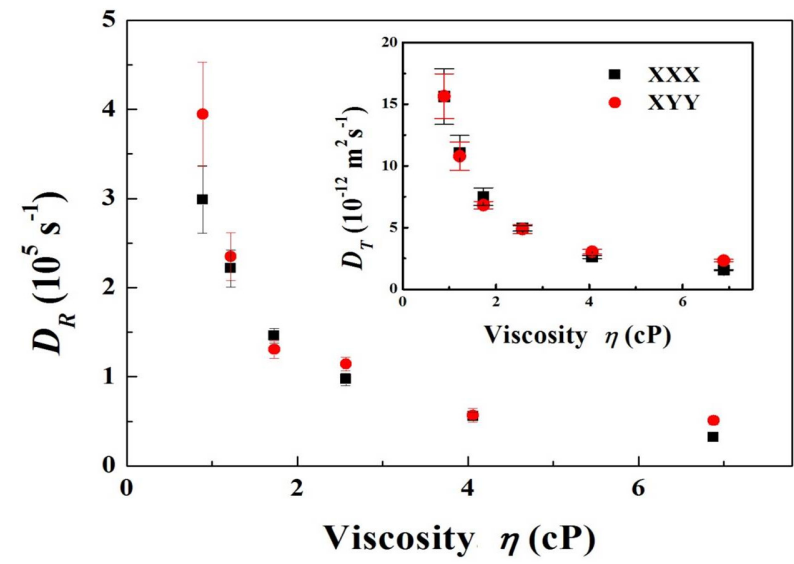

(a)

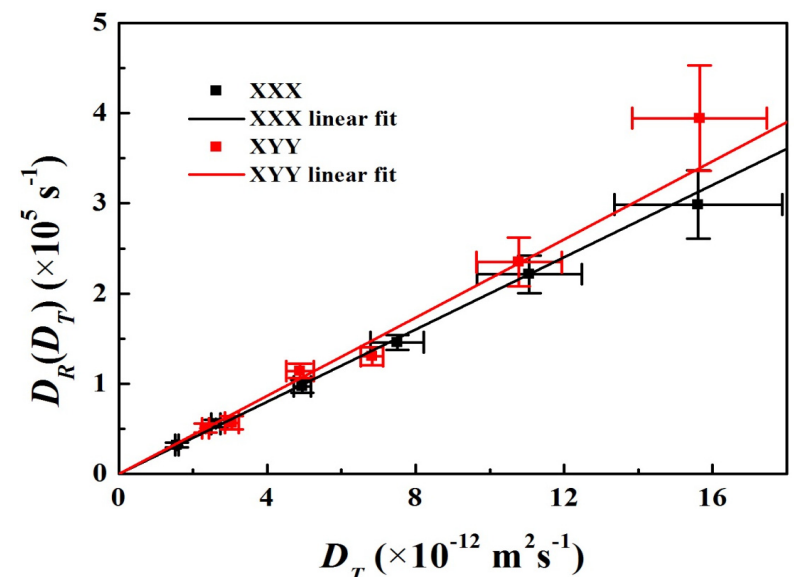

(b)

FIG. 5. (a) Effects of viscosity on translational and rotational diffusion coefficients. (b) Rotational diffusion coefficient versus translational diffusion coefficient for the $X X X$ and $X Y Y$ states.

$2.2 \times 10^{16} \mathrm{~m}^{-2}$ for the $X Y Y$ state. According to previously published theories on the diffusion coefficient [22], $D_{R} / D_{T}$ has a constant value determined by the particle's aspect ratio and the square of the particle's length. In the results herein, the linear fit with $D_{R}=0$ and $D_{T}=0$ gave a better result for the $X X X$ state than for the $X Y Y$ state.

\section{CONCLUSIONS}

We built a polarized fluorescence correlation spectroscopy system based on a confocal microscope, and measured and analyzed the correlation functions of $\mathrm{CdSe} / \mathrm{CdS}$ Q-rods in solution for various concentrations and viscosities, and for two polarization states. In our analysis of the correlation functions with respect to polarization states, translational diffusion did not depend on the polarization state, whereas rotational diffusion depended strongly on polarization. The rotational fraction was $0.75 \pm 0.05$ in the $X X X$ state and $0.26 \pm 0.03$ in the $X Y Y$ state, and the rotational diffusion time in the $X X X$ state was similar to that in the $X Y Y$ state. We determined the diffusion coefficients based on the relation between the rotational and translational diffusion coefficients. The translational and rotational diffusion coefficients respectively ranged from $1.5 \times 10^{-11} \mathrm{~m}^{2} \mathrm{~s}^{-1}$ to $2.6 \times 10^{-12} \mathrm{~m}^{2}$ $\mathrm{s}^{-1}$ and from $2.9 \times 10^{5} \mathrm{~s}^{-1}$ to $5.6 \times 10^{4} \mathrm{~s}^{-1}$. Our results can be used to determine the size of rodlike particles, or to determine the rotational diffusion coefficients of particles of other sizes [25].

\section{ACKNOWLEDGMENT}

This work was supported by a grant from the National Research Foundation of Korea (NRF), funded by the Korean Government (NRF 2012R1A1A20044140), and supported by the Priority Research Centers Program of the NRF, funded by the Ministry of Education (2009-0093818).

\section{REFERENCES}

1. J. R. Lakowicz, Principles of Fluorescence Spectroscopy, 3rd ed. (Springer Science+Business Media, 2006).

2. D. Magde, E. Elson, and W. W. Webb, "Thermodynamic fluctuations in a reacting system-measurement by fluorescence correlation spectroscopy," Phys. Rev. Lett. 29, 705-708 (1972).

3. E. L. Elson and D. Magde, "Fluorescence correlation spectroscopy. I. Conceptual basis and theory," Biopolymers 13, 1-27 (1974).

4. D. Magde, E. Elson, and W. W. Webb, "Fluorescence correlation spectroscopy. II. An experimental realization," Biopolymers 13, 29-61 (1974).

5. R. Rigler, Ü. Mets, J. Widengen, and P. Kask, "Fluorescence correlation spectroscopy with high count rate and low background: analysis of translational diffusion," Eur. Biophys. J. 22, 169-175 (1993).

6. P. Brazda, T. Szekeres, B. Bravics, K. Tóth, G. Vámosi, and L. Nagy, "Live-cell fluorescence correlation spectroscopy dissects the role of coregulator exchange and chromatin binding in retinoic acid receptor mobility," J. Cell Sci. 124, 3631-3642 (2011).

7. C. Pack, K. Saito, M. Tamura, and M. Kinjo, "Micro- 
environment and effect of energy depletion in the nucleus analyzed by mobility of multiple oligomeric EGFPs," Biophys. J. 91, 3921 (2006).

8. J. A. J. Fitzpatrick and B. F. Lillemeier, "Fluorescence correlation spectroscopy: Linking moleculear dynamics to biological function in vitro and situ," Current Opinion in Structural Biology 21, 1-11 (2011).

9. A. P. Alivisatos, "Semiconductor clusters, nanocrystals, and quantum dots," Science 271, 933-937 (1996).

10. X. Michalet, F. F. Finaud, L. A. Bentolia, J. M. Tsay, S. Doose, J. J. Li, G. Sundaresan, A. M. Wi, S. S. Gambhir, and S. Weiss, "Quantum dot for live cells, in vivo imaging, and diagnostics," Science 307, 538-544 (2005).

11. M. Ehrenberg and R. Rigler, "Rotational Brownian motion and fluorescence intensity fluctuations," Chem. Phys. 4, 390-401 (1974).

12. Ü. Mets, Fluorescence Correlation Sepctroscopy (Springer, 2001).

13. H. S. Shin, A. Okamoto, Y. Sako, S. Y. Kim, S. W. Kim, and C. G. Pack, "Characterization of the triplet state of hybridization-sensitive DNA probe by using fluorescence correlation spectroscopy," J. Phys. Chem. A 117, 27-33 (2013).

14. P. Kask, P. Piksarv, M. Pooga, Ü. Mets, and E. Lippmaa, "Separation of the rotational contribution in fluorescence correlation experiments," Biophys. J. 55, 213-220 (1989).

15. M. Ehrenberg and R. Rigler, "Fluorescence correlation spectroscopy applied to rotational diffusion of macromolecules," Q. Rev. Biophys. 9, 69-81 (1976).

16. M. Zhao, L. Jin, B. Chen, Y. Ding, H. Ma, and D. Chen, "Afterpulsing and its correction in fluorescence correlation spectroscopy experiments," Appl. Opt. 42, 4031-4036 (2003).
17. S. Deka, A. Quarta, M. G. Lupo, A. Falqui, S. Boninelli, C. Giannini, G. Morello, M. D. Giorgi, G. Lanzni, C. Spinella, R. Cinglani, T. Pellegrino, and L. Manna, "CdSe/CdS/ $\mathrm{ZnS}$ double shell nanorods with high photoluminescence efficiency and their exploitation as biolabeling probes," J. Am. Chem. Soc. 131, 2948-2958 (2009).

18. N. S. Cheng, "Formula for the viscosity of glycerol-water mixture," Ind. Eng. Chem. Res. 47, 3285-3288 (2008).

19. M. Dorfschmid, K. Müllen, A. Zumbusch, and D. Wöll, "Translational and rotational diffusion during radical bulk polymerization: A comparative investigation by full correlation fluorescence correlation spectroscopy (fcFCS)," Macromolecules 43, 6174-6179 (2010).

20. M. Müller, Indroduction to Confocal Fluorescence Microscopy, 2nd ed. (SPIE Press, Washington, USA, 2006).

21. D. S. Banks and C. Fradin, "Anomalous diffusion of proteins due to molecular crowding," Biophys. J. 89, 2060-2971 (2005).

22. J. M. Tsay, S. Doose, and S. Weiss, "Rotational and translational diffusion of peptide-coated $\mathrm{CdSe} / \mathrm{CdS} / \mathrm{ZnS}$ nanorods studied by fluorescence correlation spectroscopy," J. Am. Chem. Soc. 128, 1639-1647 (2006).

23. E. Hecht, Optics, 2nd ed. (Addison Wesley Publishing Company Inc., New York, USA, 1987).

24. A. Cooper, Biophysical Chemistry (Life Science Publishing Co., Cambridge, 2005).

25. C. Pack, H. Yukii, A. Toh-e, T. Kudo, H. Tsuchiya, A. Kaiho, E. Sakata, S. Murata, H. Yokosawa, Y. Sako, W. Baumeister, K. Tanaka, and Y. Saeki, "Quantitative live-cell imaging reveals spatio-temporal dynamics and cytoplasmic assembly of the $26 \mathrm{~S}$ proteasome," Nat. Commun. 5, 1-10 (2014). 